\title{
MicroRNA-138 suppresses cell proliferation in laryngeal squamous cell carcinoma via inhibiting EZH2 and PI3K/AKT signaling
}

\author{
FENGZHI SI, JIE SUN and CHUNLI WANG \\ Department of Otorhinolaryngology, The Second Hospital of \\ Xinjiang Medical University, Urumqi, Xinjiang 830063, P.R. China
}

Received June 16, 2016; Accepted April 11, 2017

DOI: 10.3892/etm.2017.4733

\begin{abstract}
MicroRNA (miR)-138 generally has a suppressive role in various human cancer types; however, its role and the underlying mechanisms in laryngeal squamous cell carcinoma (LSCC) have remained to be elucidated. The present study assessed the clinical significance and regulatory mechanisms of miR-138 in LSCC progression. Reverse-transcription quantitative polymerase chain reaction analysis indicated that miR-138 was significantly downregulated in LSCC tissues and cell lines. In addition, the decreased expression of miR-138 was significantly associated with poor differentiation, lymph node metastasis and advanced clinical stage of LSCC. Restoration of miR-138 expression caused a significant decrease in the proliferation of Hep-2 LSCC cells, while knockdown of miR-138 significantly promoted Hep-2 cell proliferation. A luciferase reporter assay further identified enhancer of zeste homologue 2 (EZH2) as a direct target gene of miR-138, and the protein expression of EZH2 was negatively regulated by miR-138 in Hep- 2 cells. Furthermore, overexpression of EZH2 eliminated the suppressive effects of miR-138 on Hep-2 cell proliferation via activation of phosphoinositide-3 kinase (PI3K)/AKT signaling. In addition, EZH2 was found to be significantly upregulated in LSCC tissues and to be inversely correlated to the miR-138 levels. The results of the present study demonstrated that miR-138 inhibits the proliferation of LSCC cells, at least partly via targeting EZH2 and inhibiting PI3 K/AKT signaling. The present study highlighted the clinical significance of the miR-138/EZH2 axis in LSCC.
\end{abstract}

Correspondence to: Professor Chunli Wang, Department of Otorhinolaryngology, The Second Hospital of Xinjiang Medical University, 38 North Two Lane of South Lake East Road, Urumqi, Xinjiang 830063, P.R. China

E-mail: xiangyawangcl@sina.com

Key words: laryngeal squamous cell carcinoma, microRNA, proliferation, enhancer of zeste homologue 2

\section{Introduction}

Laryngeal squamous cell carcinoma (LSCC) is a common malignant tumor type of the otolaryngeal region, accounting for $1-2 \%$ of all human cancer cases worldwide $(1,2)$. Although great efforts have been made regarding tumor diagnosis and treatment, the prognosis of patients with LSCC still remains unsatisfactory (1-3). Investigation of the regulatory mechanisms underlying LSCC development and progression may lead to the development of effective therapeutic strategies for LSCC.

MicroRNAs (miRs), a class of of short non-coding RNAs, may cause the degradation or translational inhibition of their target mRNAs via directly binding to their 3'-untranslated region (UTR) (4,5). A large number of miRs have been found to be involved in various biological processes, including cell survival, apoptosis, proliferation, differentiation, cell cycle progression and migration $(6,7)$. Deregulation of miRs has been observed in a variety of human cancer type $(8,9)$. Certain specific miRs act as key regulators of the expression of oncogenes or tumor suppressors in LSCC, and may thus be utilized as potential therapeutic targets for this disease $(10,11)$. For instance, miR-144 suppresses the growth and metastasis of LSCC by targeting insulin receptor substrate 1 (10), while miR-365a promotes LSCC growth and metastasis by activating the phosphoinositide-3 kinase (PI3K)/AKT signaling pathway (11). However, the roles of miRs in the development and progression of LSCC as well as the underlying mechanisms remains to be fully elucidated.

miR-138 has been reported to have a suppressive role in certain common types of human cancer (12-14). For instance, Jiang et al (12) found that miR-138 has a suppressive role in osteosarcoma by targeting differentiated embryonic chondrocyte gene 2. Wei et al (13) reported that miR-138 exerts anti-glioma efficacy by targeting immune checkpoints. Furthermore, miR-138 was reported to inhibit the proliferation of cervical cancer cells by targeting c-Met (14). Recently, miR-138 was found to be significantly downregulated in LSCC tissues compared with that in paired normal laryngeal tissues, and miR-138 inhibit the invasion of LSCC cells by directly targeting zinc finger E-box-binding homeobox 2, suggesting that miR-138 may have suppressive effects on 
LSCC metastasis (15). However, the exact role of miR-138 in LSCC growth has remained to be fully elucidated.

Therefore, the present study aimed to investigate the clinical significance of miR-138 in LSCC and the regulatory mechanism of miR-138 in LSCC cell proliferation.

\section{Materials and methods}

Tissue collection. The present study was approved by the Ethic Committee of the Second Hospital of Xinjiang Medical University (Urumqi, China). A total of 65 LSCC tissues and their matched adjacent non-tumorous tissues were collected between March 2012 and April 2015 at the Second Hospital of Xinjiang Medical University. Written informed consent was obtained from all patients included. None of the patients received any radiation therapy or chemotherapy prior to surgical resection. The tissue samples were immediately snap-frozen in liquid nitrogen after surgical resection and stored at $-80^{\circ} \mathrm{C}$ until use. The clinical information of patients included in this study is summarized in Table I.

Reverse transcription-quantitative polymerase chain reaction $(R T-q P C R)$. Total RNA was extracted using TRIzol reagent (Thermo Fisher Scientific, Inc., Waltham, MA, USA), and $1 \mu \mathrm{g}$ of RNA was reverse transcribed into complementary (c) DNA using a PrimeScript 1st Strand cDNA Synthesis kit (Takara, Otsu, Japan) according to the manufacturer's instructions. For miR-138 expression detection, real-time PCR was performed using a miRNA Q-PCR Detection kit (GeneCopoeia Rockville, MD, USA) on an ABI 7500 thermocyeler (Thermo Fisher Scientific, Inc.), according to the manufacturer's instructions. U6 gene was used as an internal control (cat. no. HmiRQP9001; Guangzhou Fulengen Co., Ltd., Guangzhou, China; sequence not provided). For mRNA expression detection, qPCR was performed using a SYBR-Green I Real-Time PCR kit (Biomics, Nantong, China) according to the manufacturer's instructions. GAPDH was used as an internal control. The specific primer pairs were as follows: Enhancer of zeste homologue 2 (EZH2) forward, 5'-AATCAGAGTACATGC GACTGAGA-3' and reverse, 5'-GCTGTATCCTTCGCTGTT TCC-3'; GAPDH forward, 5'-CTGGGCTACACTGAGCAC C-3' and reverse, 5'-AAGTGGTCGTTGAGGGCAATG-3'. The reaction conditions were $95^{\circ} \mathrm{C}$ for $5 \mathrm{~min}$, followed by 40 cycles of denaturation at $95^{\circ} \mathrm{C}$ for $15 \mathrm{sec}$ and an annealing/elongation step at $60^{\circ} \mathrm{C}$ for $30 \mathrm{sec}$. The relative expression was analyzed by using the $2^{-\Delta \Delta \mathrm{Cq}}$ method (16).

Cell culture and transfection. The Hep-2 human LSCC cell line was purchased from the Cell Bank of the Chinese Academy of Sciences (Shanghai, China). Cells were cultured in Dulbecco's modified Eagle's medium (Thermo Fisher Scientific, Inc.) with $10 \%$ fetal bovine serum (Thermo Fisher Scientific, Inc.) at $37^{\circ} \mathrm{C}$ in a humidified atmosphere containing $5 \% \mathrm{CO}_{2}$.

Cell transfection. Cells were transfected with scrambled miR mimics (miR-NC), miR-138 mimics, NC inhibitor or miR-138 inhibitor, or co-transfected with miR-138 mimics and pcDNA3.1-EZH2 expression plasmid (all purchased from Yearthbio, Changsha, China) by using Lipofectamine ${ }^{\circledR} 2000$
(Thermo Fisher Scientific, Inc.) according to the manufacturer's instructions.

Western blot analysis. Cells were lysed with ice-cold lysis buffer (Thermo Fisher Scientific, Inc.), and protein concentrations were determined using a Pierce BCA Protein Assay kit (Thermo Fisher Scientific, Inc.), according to the manufacturer's protocol. Proteins (50 $\mu \mathrm{g}$ per lane) were separated by $10 \%$ SDS-PAGE, followed by transfer onto a polyvinylidene difluoride membrane (Thermo Fisher Scientific, Inc.). The membrane was then incubated with PBS containing 5\% milk at room temperature for $3 \mathrm{~h}$, followed by incubation with the rabbit anti-EZH2 primary antibody (1:100; ab186006; Abcam, Cambridge, MA, USA), rabbit anti-PI3K primary antibody (1:100; ab32569; Abcam), rabbit anti-p-PI3K primary antibody (1:100; ab182651; Abcam), rabbit anti-AKT primary antibody (1:50; ab28422; Abcam), and rabbit anti-p-AKT primary antibody (1:100; ab81283; Abcam) at room temperature for $3 \mathrm{~h}$. After washing with PBS for 3 times, the membrane was incubated with goat anti-rabbit secondary antibody (1:5,000; ab6721; Abcam) at room temperature for $40 \mathrm{~min}$. After washing with PBS for 3 times, a Super Signal West Pico Chemiluminescent Substrate kit (Pierce; Thermo Fisher Scientific, Inc.) was used to detect signals on X-ray film according to the manufacturer's instructions. The relative protein expression was presented as the density ratio vs. GAPDH.

MTT assay. Hep- 2 cell suspension $\left(5 \times 10^{4}\right.$ cells/well $)$ was seeded in a 96-well plate and cultured for $0,24,48$ or $72 \mathrm{~h}$. MTT $(10 \mu l, 5 \mathrm{mg} / \mathrm{ml}$, Thermo Fisher Scientific, Inc.) was then added to each well, followed by incubation at $37^{\circ} \mathrm{C}$ for $4 \mathrm{~h}$. The supernatant was removed and $100 \mu 1$ dimethyl sulfoxide was added to each well. The absorbance at $570 \mathrm{~nm}$ was determined using a Model 680 Microplate Reader (Bio-Rad Laboratories, Inc., Hercules, CA, USA).

Bioinformatics predication and luciferase reporter assay. Targetscan online software (http://www.targetscan.org) was used to predicate the putative targets of miR-138. The mutant type (MT) of the 3'UTR fragment of EZH2 lacking complementarity with the miR-138 seed sequence was generated using the QuickChange Site-Directed Mutagenesis kit (Stratagene, La Jolla, CA, USA), according to the manufacturer's instructions. The wild-type (WT) or MT EZH2 3'UTR fragment was cloned into the downstream region of the firefly luciferase coding region of the pMIR-GLO ${ }^{\text {TM }}$ luciferase vector (Promega Corp., Madison, WI, USA). Hep-2 cells were co-transfected with the WT-EZH2-3'UTR or MT-EZH2-3'UTR plasmid, and miR-NC or miR-138 mimics. The luciferase activity was detected after transfection for $48 \mathrm{~h}$ using the Dual Luciferase Reporter Assay system (Promega Corp.), according to the manufacturer's instructions.

Statistical analysis. Values are expressed as the mean \pm standard deviation of three independent experiments. Differences between two groups were analyzed using Student's t-test. The association between miR-138 expression and clinical characteristics of LSCC patients were analyzed using the chi-square test. Pearson correlation analysis was used to analyze the 
Table I. Association between miR-138 expression and clinicopathological characteristics of patients with laryngeal squamous cell carcinoma.

\begin{tabular}{|c|c|c|c|c|}
\hline Variable & Number & Low miR-138 & High miR-138 & P-value \\
\hline Age (years) & & & & 0.457 \\
\hline$<55$ & 24 & 14 & 10 & \\
\hline$\geq 55$ & 41 & 20 & 21 & \\
\hline Sex & & & & 0.478 \\
\hline Male & 39 & 19 & 20 & \\
\hline Female & 26 & 15 & 11 & \\
\hline Differentiation & & & & 0.018 \\
\hline Well/moderate & 43 & 18 & 25 & \\
\hline Poor & 22 & 16 & 6 & \\
\hline Lymph node metastasis & & & & 0.039 \\
\hline No & 42 & 18 & 24 & \\
\hline Yes & 23 & 16 & & \\
\hline Distant metastasis & & & & 0.099 \\
\hline No & 56 & 27 & 29 & \\
\hline Yes & 9 & 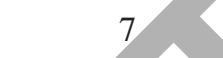 & 2 & \\
\hline Clinical stage & & & & 0.017 \\
\hline $\mathrm{I} / \mathrm{II}$ & 34 & 13 & 21 & \\
\hline III/IV & 31 & 21 & 10 & \\
\hline
\end{tabular}

correlation between miR-138 and EZH2 expression in LSCC tissues. SPSS 19.0 (International Business Machines Corp., Armonk, NY, USA) was used to perform statistical analysis. $\mathrm{P}<0.05$ was considered to indicate a statistically significant difference.

\section{Results}

miR-138 is downregulated in LSCC tissues and significantly associated with LSCC progression. RT-qPCR was used to examine the miR-138 expression in LSCC tissues and paired adjacent non-tumorous tissues. As shown in Fig. 1, the miR-138 levels were significantly decreased in LSCC tissues compared with those in adjacent non-tumorous tissues. The clinical significance of miR-138 in LSCC was then assessed. Using the mean value of the miR-138 expression as a cutoff point, the patients enrolled in the present study were divided into two groups with regard to high and low miR-138 expression. It was revealed that low miR-138 levels were significantly associated with poor differentiation, lymph node metastasis and advanced clinical stage, but not associated with age, sex or distant metastasis (Table I). Accordingly, the present findings suggested that decreased expression of miR-138 is associated with LSCC progression.

miR-138 has inhibitory effects on LSCC cell proliferation. As miR-138 was significantly downregulated in LSCC tissues, LSCC Hep-2 cells were transfected with miR-138 mimics to restore its expression. As indicated in Fig. 2A, transfection with miR-138 mimics significantly increased the miR-138

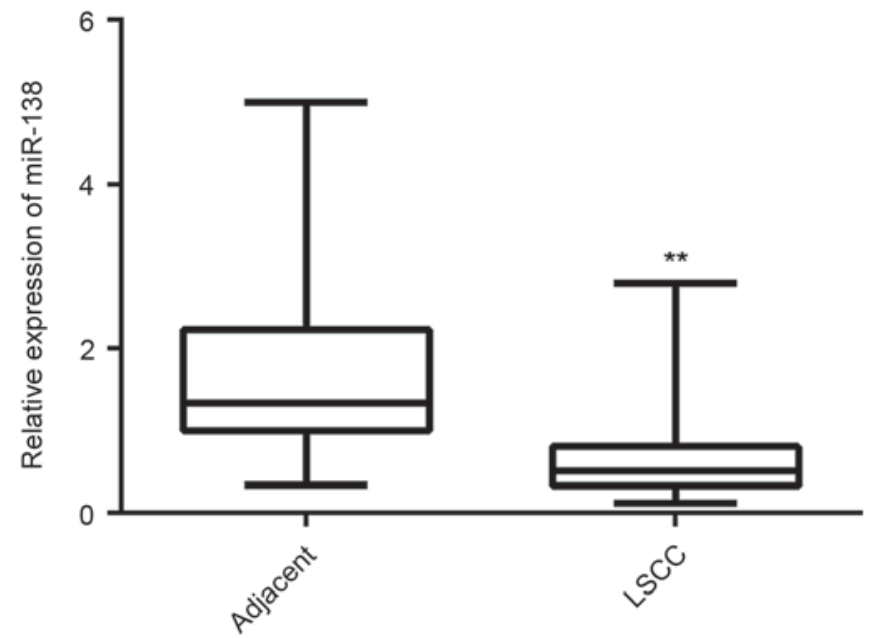

Figure 1. Reverse transcription-quantitative polymerase chain reaction was performed to examine the miR-138 expression in LSCC tissues compared to that in adjacent non-tumorous tissues. ${ }^{* *} \mathrm{P}<0.01$ vs. Adjacent. miR, microRNA; LSCC, laryngeal squamous cell carcinoma.

levels compared with those in the control group (Fig. 2A), while transfection with miR-NC did not affect the miR-138 levels in Hep-2 cells (Fig. 2A). The MTT assay revealed that restoration of miR-138 expression caused a significant decrease in the proliferation of Hep- 2 cells, when compared with that in the control group (Fig. 2B). To confirm these findings, Hep-2 cells were then transfected with miR-138 inhibitor to further knockdown its expression. As shown in 
A
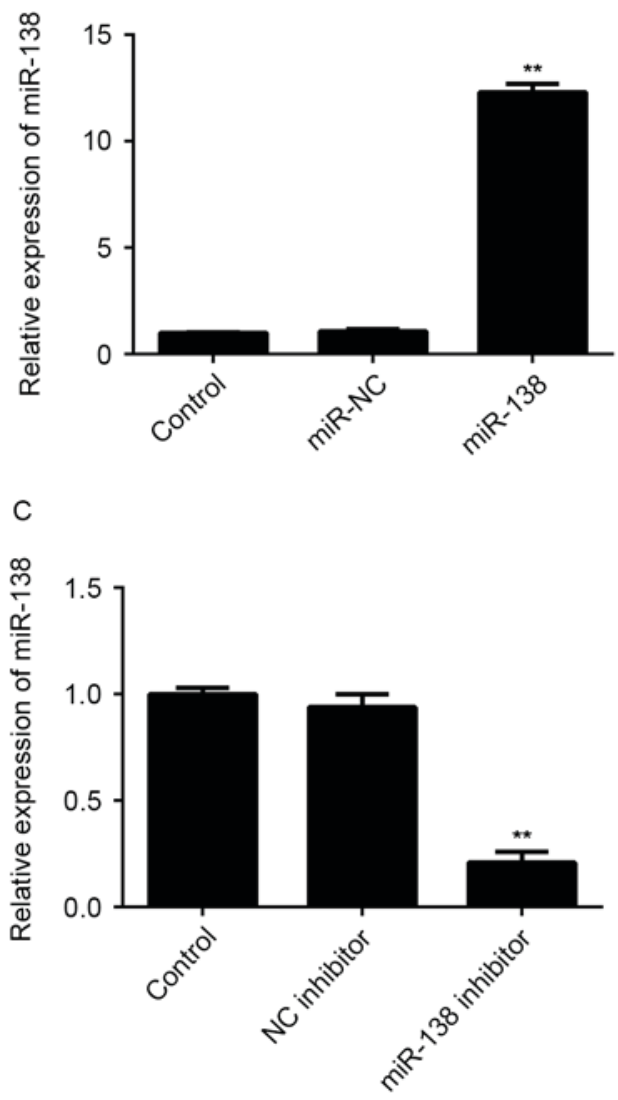

B

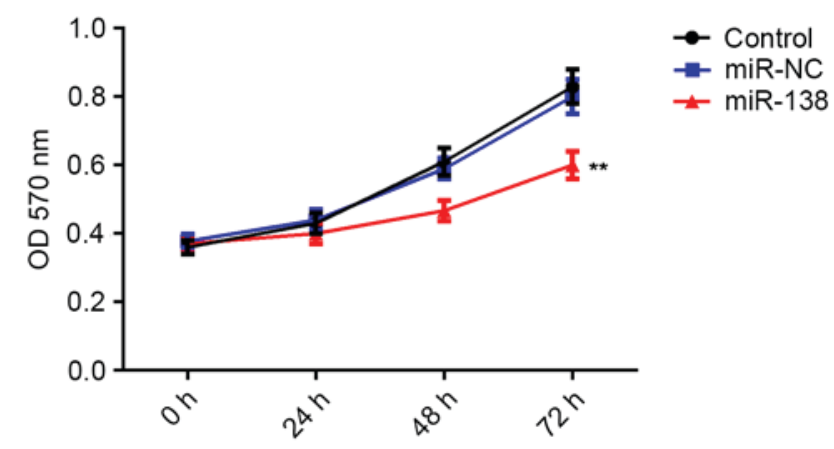

D

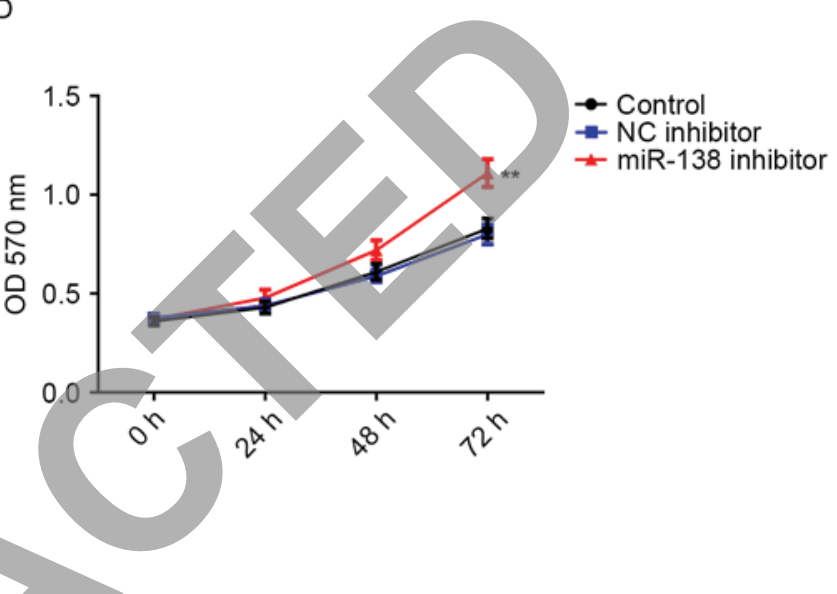

Figure 2. (A) RT-qPCR was performed to examine the miR-138 expression and (B) an MTT assay was performed to determine cell proliferation in Hep-2 cells transfected with miR-138 mimics or miR-NC. (C) RT-qPCR was performed to examine the miR-138 expression and (D) an MTT was performed to determine cell proliferation in Hep-2 cells transfected with miR-138 inhibitor or NC inhibitor. Non-transfected Hep-2 cells were used as a Control. ** P $<0.01$ vs. Control. miR, microRNA; NC, negative control; miR-NC, seramble miR mimies; RT-qPCR, reverse transcription-quantitative polymerase chain reaction; OD, optical density.

Fig. 2C, transfection with miR-138 inhibitor significantly reduced the miR-138 levels in Hep- 2 cells compared with those in the control group, while transfection with $\mathrm{NC}$ inhibitor did not. An MTT assay further indicated that knockdown of miR-138 promoted the proliferation of Hep-2 cells (Fig. 2D). Therefore, the results suggested that miR-138 has inhibitory effects on LSCC cell proliferation.

EZH2 is a target gene of miR-138 in LSCC cells. To further study the mechanism of by which miR-138 regulates LSCC cell proliferation, the putative target genes of miR-138 were predicated using Targetscan software. As shown in Fig. 3A and B, EZH2 was predicated to be a target of miR-138 and their association was evolutionarily conserved. To confirm this direct regulatory interaction in vitro, the WT or MT fragment of the EZH2 3'UTR was inserted downstream of the firefly luciferase coding region of pMIR-GLO ${ }^{\mathrm{TM}}$ luciferase vector, generating WT-EZH2-3'UTR or MT-EZH2-3'UTR luciferase reporter plasmid, respectively (Fig. 3C and D). Hep-2 cells were then co-transfected with WT-EZH2-3'UTR or MT-EZH2-3'UTR plasmid, and miR-NC or miR-138 mimic, respectively. The luciferase activity was significantly decreased in Hep-2 cells co-transfected with miR-138 mimics and WT-EZH2-3'UTR plasmid, but remained unchanged in cells co-transfected with miR-138 mimics and MT-EZH2-3'UTR plasmid, when compared with that in the control group (Fig. 3E). Accordingly, miR-138 directly binds to the 3'UTR of EZH2 mRNA in Hep-2 cells.

The present study further assessed the regulatory role of miR-138 in EZH2 expression in LSCC cells. Western blot analysis was performed to detect the protein levels of EZH2 in Hep-2 cells after overexpression or downregulation of miR-138. As indicated in Fig. 4A, overexpression of miR-138 significantly decreased the EZH2 protein expression. By contrast, inhibition of miR-138 led to an upregulation of EZH2 protein levels in Hep-2 cells (Fig. 4B). Therefore, EZH2, as a direct target gene of miR-138, is negatively regulated by miR-138 in Hep-2 cells.

Overexpression of EZH2 eliminates the suppressive effects of miR-138 on LSCC cell proliferation by activating $P I 3 K / A K T$ signaling. To investigate whether EZH2 was involved in the miR-138-mediated proliferation of Hep-2 cells, miR-138-overexpressing Hep-2 cells were further transfected with pcDNA3.1-EZH2 open reading frame plasmid to reverse the suppressive effect of miR-138 overexpression on EZH2 expression. After transfection, the protein levels of EZH2 were significantly upregulated (Fig. 5A). MTT assay data further indicated that the proliferative capacity of Hep-2 cells was significantly higher in the miR-138 + EZH2 group 


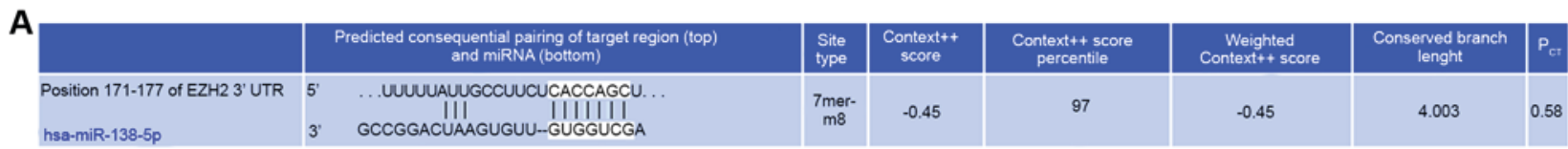

B

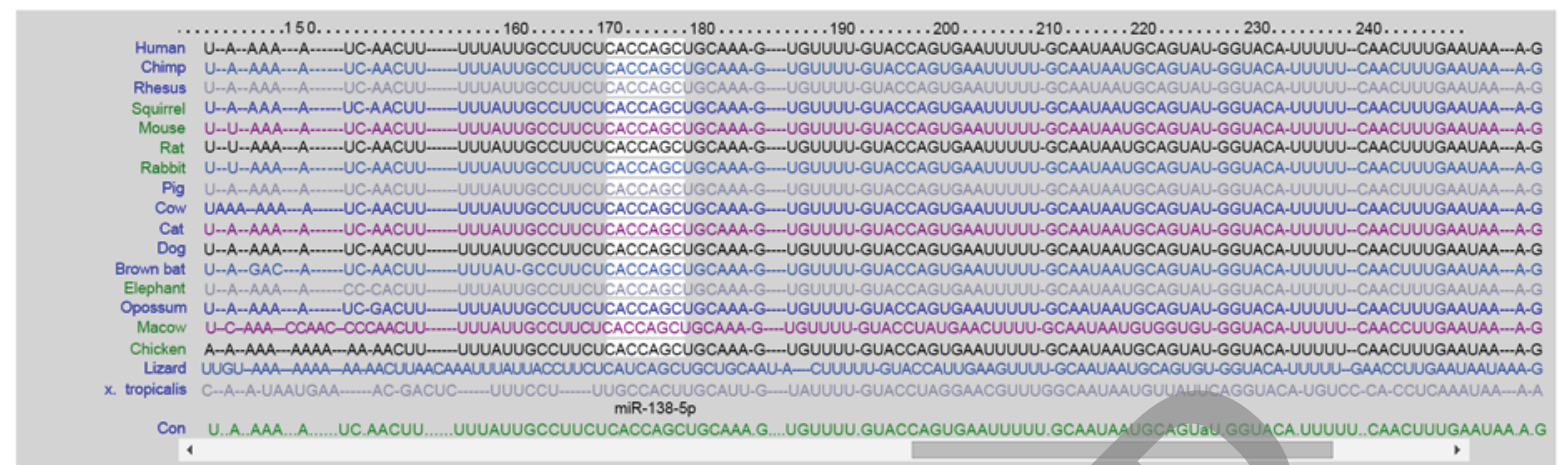

C

D

WT EZH2 3' UTR 5'-...GCCUUCUCACCAGCU...-3'

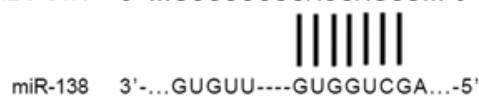

MT EZH2 3' UTR 5'-... GCCUUCUGUGGUCGU...-3'

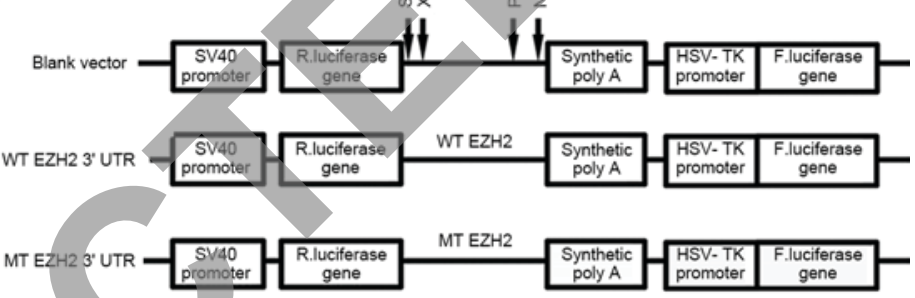

E

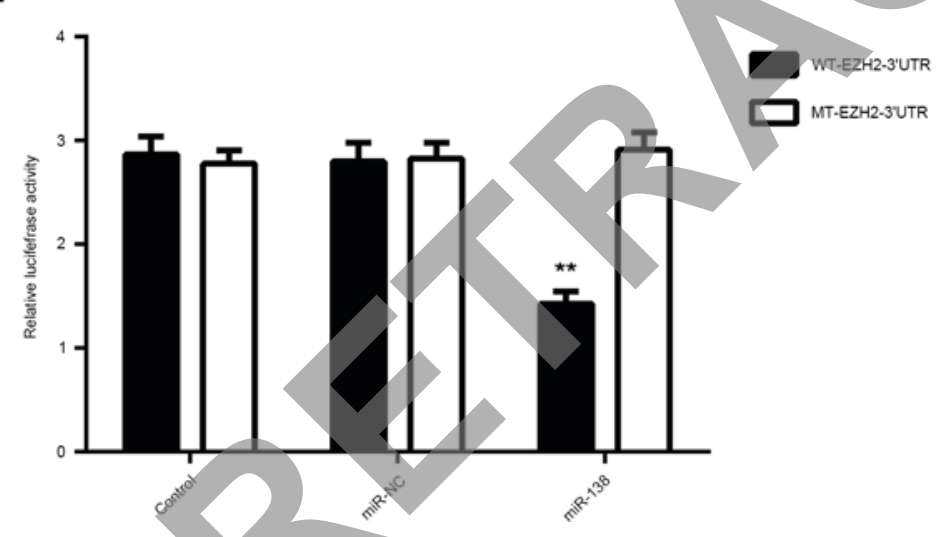

Figure 3. (A and B) Targetscan software indicated that EZH2 was a target gene of miR-138 and their association was evolutionally conserved. (C and D) To confirm this targeting association, luciferase reporter vectors containing a WT or MT sequence of the EZH2 3'-UTR were generated. (E) The luciferase activity was significantly reduced in Hep-2 cells co-transfected with the WT-EZH2 luciferase reporter vector and miR-138 mimics, when compared to that in the control group, which was eliminated by transfection with MT-EZH2 luciferase reporter vector. ${ }^{* *}$ P $<0.01$ vs. Control. miR, microRNA; UTR, untranslated region, WT, wild-type; MT, mutant type; EZH2, enhancer of zeste homologue 2.

compared with that in the miR-138 group, indicating that overexpression of EZH2 reversed the suppressive effects of miR-138 on Hep-2 cell proliferation (Fig. 5B). Subsequently, the activity of PI3 K/AKT, a key signaling pathway involved in cell proliferation, was examined. As shown in Fig. 5C and D, the activity of PI3K and AKT was significantly higher in the miR-138 + EZH2 group compared with that in the miR-138 group. According to these results, it is suggested that miR-138 inhibits LSCC cell proliferation via inhibition of EZH2 expression and PI3K/AKT signaling.

EZH2 is upregulated in LSCC and inversely correlated with miR-138 expression. Finally, the expression of EZH2 in LSCC tissues and adjacent non-tumor tissues was detected. RT-qPCR demonstrated that the EZH2 mRNA levels were significantly increased in LSCC tissues compared with those in adjacent non-tumor tissues (Fig. 6A). In addition, the EZH2 levels were inversely correlated to the miR-138 levels in LSCC tissues (Fig. 6B). Therefore, the upregulation of EZH2 may be caused by the decreased miR-138 levels in LSCC tissues, which promotes LSCC progression.

\section{Discussion}

The regulatory role of miR-138 in LSCC growth and the underlying mechanisms have remained elusive. In the present study, RT-qPCR results indicated that miR-138 was significantly downregulated in LSCC tissues. In addition, 

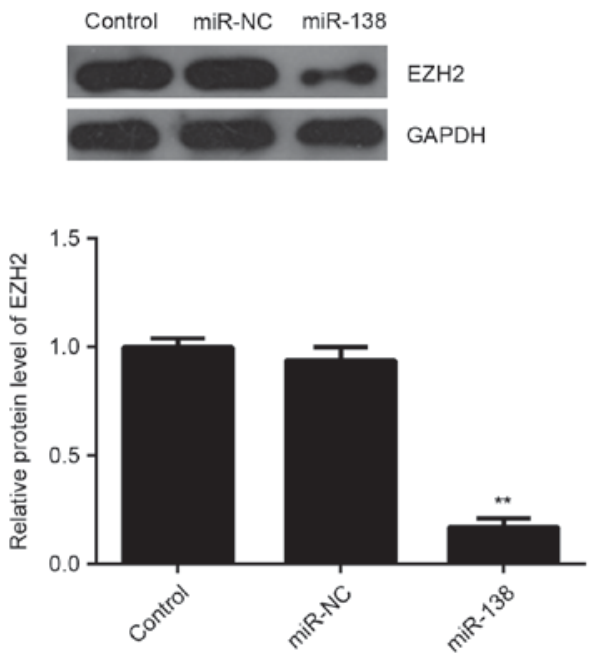
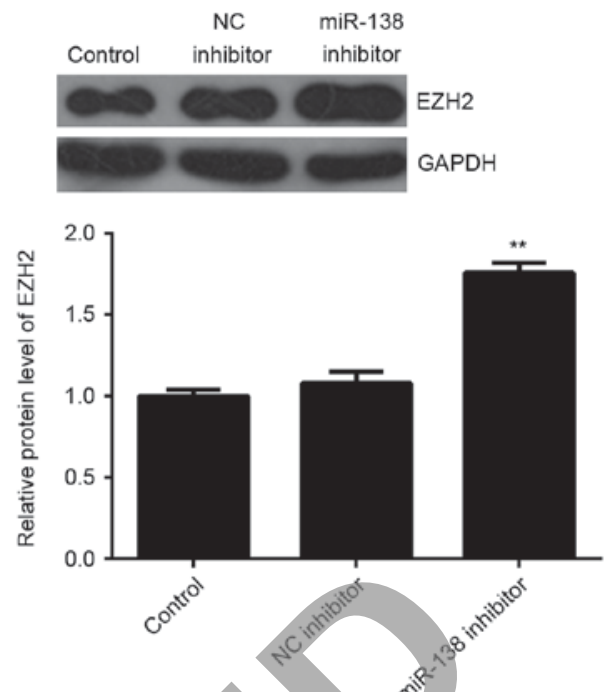

Figure 4. (A) Western blot analysis was used to examine the protein expression of EZH2 in Hep-2 cells transfected with miR-138 inhibitor or NC inhibitor. (B) Western blot was used to examine the protein expression of EZH2 in Hep-2 cells transfected with miR-138 inhibitor or NC inhibitor. Non-transfected Hep-2 cells were used as a Control. ${ }^{* *} \mathrm{P}<0.01$ vs. Control. EZH2, enhancer of zeste homologue 2; miR, microRNA; NC, negative control.

A
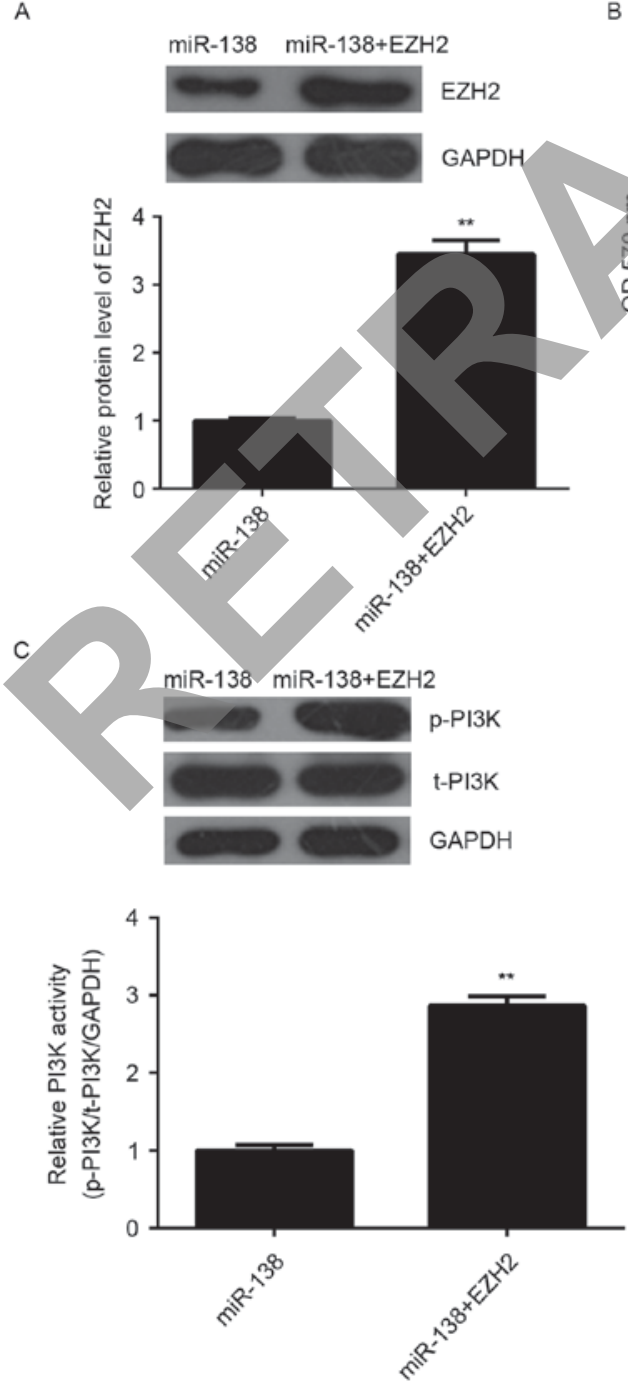

$B$

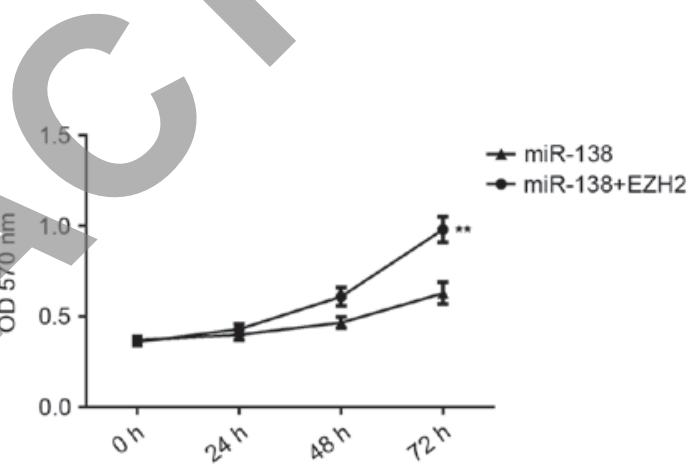

D miR-138 miR-138+EZH2
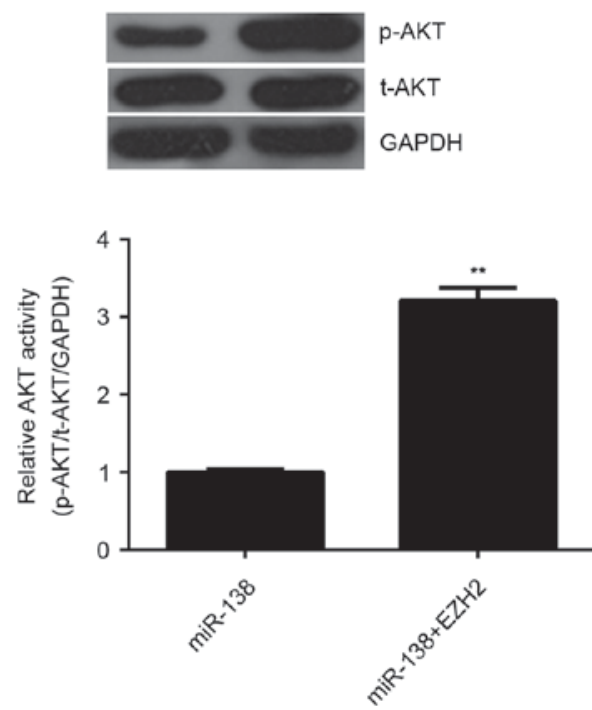

Figure 5. Hep-2 cells were transfected with miR-138 mimics or co-transfected with miR-138 mimics and EZH2 expression plasmid. (A) Western blot analysis was performed to examine the protein expression of EZH2. (B) An MTT assay was used to examine cell proliferation. (C and D) Western blot analysis was performed to examine the protein levels of p-PI3K, t-PI3K, p-AKT and t-AKT. The relative activity of PI3K and AKT was determined. ${ }^{* *}<0.01$ vs. miR-138. miR, microRNA; p-PI3 K, phosphorylated phosphoinositide-3 kinase; t-AKT, total AKT; OD, optical density; EZH2, enhancer of zeste homologue 2. 
A

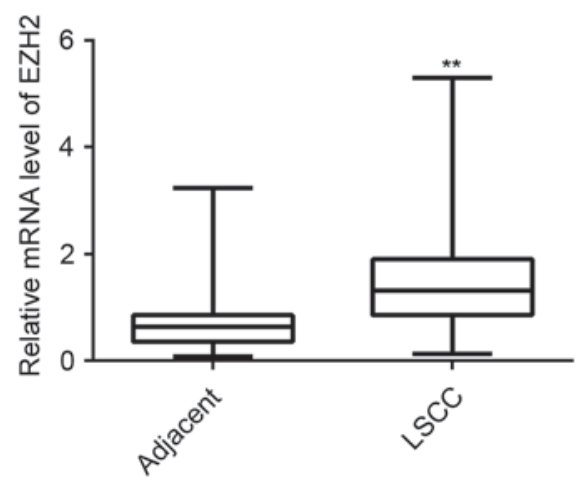

B

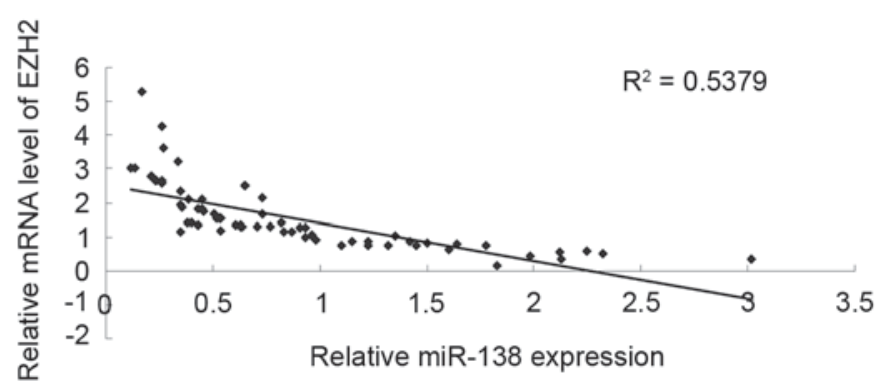

Figure 6. (A) Reverse transcription-quantitative polymerase chain reaction was performed to examine the mRNA of EZH2 in LSCC tissues compared with that in adjacent non-tumorous tissues. ${ }^{* *} \mathrm{P}<0.01$ vs. Adjacent. (B) An inverse correlation was observed between the miR-138 expression and the mRNA expression of EZH2 in LSCC tissues ( $\mathrm{P}<0.01)$. miR, microRNA; LSCC, laryngeal squamous cell carcinoma; EZH2, enhancer of zeste homologue 2.

the decreased expression of miR-138 was significantly associated with poor differentiation, lymph node metastasis and advanced clinical stage of LSCC. Restoration of miR-138 expression caused a significant decrease in the proliferation of Hep-2 LSCC cells, while knockdown of miR-138 significantly promoted Hep-2 cell proliferation. A luciferase reporter assay further identified EZH2 as a direct target gene of miR-138, and the protein expression of EZH2 was found to be negatively regulated by miR-138 in Hep-2 cells. Furthermore, overexpression of EZH2 eliminated the suppressive effects of miR-138 on Hep-2 cell proliferation via activation of PI3 K/AKT signaling. In addition, EZH2 was found to be significantly upregulated in LSCC tissues and to be inversely correlated to the miR-138 levels.

miR-138 has been found to generally act as a tumor suppressor in certain common human cancer types by targeting different oncogenes $(17,18)$. For instance, Yeh et al (19) found that miR-138 suppressed the invasion and metastasis of ovarian cancer cells by targeting SRY-box 4 and hypoxia-inducible factor-1 $\alpha$. Ye et al (20) reported that $\mathrm{miR}-138$ inhibited the proliferation of non-small cell lung cancer (NSCLC) cells by targeting 3-phosphoinositide-dependent protein kinase-1. Xu et al (21) found that miR-138 suppressed the proliferation of oral squamous cell carcinoma cells by targeting Yes-associated protein 1. It was also reported to suppress invasion and promotes apoptosis in head and neck squamous cell carcinoma cells (22). The present study found that miR-138 was significantly decreased in LSCC tissues. Furthermore, it was demonstrated that the decreased expression of miR-138 was significantly associated with the malignant progression of LSCC. Consistent with these findings, Gao et al (15) reported that miR-138 was downregulated in LSCC tissues compared with that in paired normal laryngeal tissue. In addition, they found that miR-138 inhibited the invasion of LSCC cells (15). However, the exact role of miR-138 in regulating LSCC cell proliferation has remained to be fully elucidated. The present study demonstrated that miR-138 had suppressive effects on Hep-2 cell proliferation.

The histone methyltransferase EZH2 is the catalytic subunit of polycomb repressive complex 2, a highly conserved protein complex that regulates gene expression by methylating lysine 27 on histone H3 (23). EZH2 has also been demonstrated to have a promoting role in various human cancer types and may thus become a potential therapeutic target for cancer treatment. For instance, EZH2 was significantly upregulated in luminal A breast cancer and the increased EZH2 levels were associated with poor overall survival (24). Furthermore, EZH2 promotes the proliferation of colorectal cancer stem-like cells by activation of $\mathrm{p} 21$ cip1-Wnt/ $\beta$-catenin signaling (25). Recently, Yu et al (26) found that knockdown of EZH2 inhibited LSCC cell proliferation and suppressed PI3K/AKT activation in LSCC cells. The present study demonstrated that EZH2 was a direct target of miR-138 and its protein expression was negatively regulated by miR-138 in Hep-2 cells. In fact, the targeting association between miR-138 and EZH2 was also reported in several other cancer types, including NSCLC, glioblastoma, clear cell renal cell carcinoma and osteosarcoma (27-30). Therefore, the present findings expand the understanding of the miR-138/EZH2 axis in human cancers. Furthermore, it was demonstrated that overexpression of EZH2 eliminated the suppressive effects of miR-138 on cell proliferation in Hep-2 cells, accompanied with upregulation of PI3K/AKT signaling, suggesting that miR-138 inhibits the proliferation of LSCC cells via targeting EZH2 and thus suppressing PI3K/AKT signaling. In addition, it was found that the EZH2 levels were significantly upregulated in LSCC tissues compared with those in their paired non-tumorous tissues, and an inverse correlation was identified between the miR-138 and EZH2 levels in LSCC tissues, suggesting that the increased EZH2 expression may be due to the downregulation of miR-138 in LSCC, which further promoted the malignant progression of this disease. Similarly, another miR, miR-340, was also found to inhibit LSCC cell proliferation via inhibiting the expression of EZH2 (26). Accordingly, EZH2 may be a key target of miRs in human cancer.

In conclusion, the present study suggested that miR-138 was significantly downregulated in LSCC and has a suppressive role in LSCC cell proliferation through inhibiting the EZH2 expression and PI3K/AKT signaling. Therefore, the miR-138/EZH2 interaction may become a useful therapeutic target for the treatment of LSCC. 


\section{References}

1. Siegel RL, Miller KD and Jemal A: Cancer statistics, 2015. CA Cancer J Clin 65: 5-29, 2015

2. Torre LA, Bray F, Siegel RL, Ferlay J, Lortet-Tieulent J and Jemal A: Global cancer statistics, 2012. CA Cancer J Clin 65 87-108, 2015.

3. Lionello M, Staffieri A and Marioni G: Potential prognostic and therapeutic role for angiogenesis markers in laryngeal carcinoma. Acta Otolaryngol 132: 574-582, 2012.

4. Ambros V: The functions of animal microRNAs. Nature 431: 350-355, 2004

5. Calin GA and Croce CM: MicroRNA signatures in human cancers. Nat Rev Cancer 6: 857-866, 2006.

6. Croce CM and Calin GA: miRNAs, cancer, and stem cell division. Cell 122: 6-7, 2005

7. Bartel DP: MicroRNAs: Genomics, biogenesis, mechanism, and function. Cell 116: 281-297, 2004.

8. Mannavola F, Tucci M, Felici C, Stucci S and Silvestris F: miRNAs in melanoma: A defined role in tumor progression and metastasis. Expert Rev Clin Immunol 12: 79-89, 2016.

9. Wen Y, Han J, Chen J, Dong J, Xia Y, Liu J, Jiang Y, Dai J, Lu J, Jin G, et al: Plasma miRNAs as early biomarkers for detecting hepatocellular carcinoma. Int J Cancer 137: 1679-1690, 2015.

10. Wu X, Cui CL, Chen WL, Fu ZY, Cui XY and Gong X: miR-144 suppresses the growth and metastasis of laryngeal squamous cell carcinoma by targeting IRS1. Am J Transl Res 8: $1-11,2016$.

11. Geng J, Liu Y, Jin Y, Tai J, Zhang J, Xiao X, Chu P, Yu Y, Wang SC, Lu J, et al: MicroRNA-365a-3p promotes tumor growth and metastasis in laryngeal squamous cell carcinoma. Oncol Rep 35: 2017-2026, 2016.

12. Jiang B, Mu W, Wang J, Lu J, Jiang S, Li L, Xu H and Tian H: MicroRNA-138 functions as a tumor suppressor in osteosarcoma by targeting differentiated embryonic chondrocyte gene 2 . J Exp Clin Cancer Res 35: 69, 2016.

13. Wei J, Nduom EK, Kong LY, Hashimoto Y, Xu S, Gabrusiewicz K Ling X, Huang N, Qiao W, Zhou S, et al: MiR-138 exerts anti-glioma efficacy by targeting immune checkpoints. Neuro Oncol 18: 639-648, 2016.

14. Li B, Yang XX, Wang D and Ji HK: MicroRNA-138 inhỉbits proliferation of cervical cancer cells by targeting c-Met. Eur Rev Med Pharmacol Sci 20: 1109-1114,2016.

15. Gao S, Wang J, Xie J, Zhang T and Dong P: Role of miR-138 in the regulation of larynx carcinoma cell metastases. Tumour Biol: Oct 24, 2015 (Epub ahead of print).

16. Livak KJ and Schmittgen TD: Analysis of relative gene expression data using real-time quantitative PCR and the 2(-Delta Delta C(T)) method. Methods 25: 402-408, 2001.
17. Wang W, Zhao LJ, Tan YX, Ren H and Qi ZT: MiR-138 induces cell cycle arrest by targeting cyclin D3 in hepatocellular carcinoma. Carcinogenesis 33: 1113-1120, 2012.

18. Song T, Zhang X, Wang C, Wu Y, Cai W, Gao J and Hong B MiR-138 suppresses expression of hypoxia-inducible factor $1 \alpha$ (HIF-1 $\alpha$ ) in clear cell renal cell carcinoma 786-O cells. Asian Pac J Cancer Prev 12: 1307-1311, 2011.

19. Yeh YM, Chuang CM, Chao KC and Wang LH: MicroRNA-138 suppresses ovarian cancer cell invasion and metastasis by targeting SOX4 and HIF-1 $\alpha$. Int J Cancer 133: 867-878, 2013.

20. Ye XW, Yu H, Jin YK, Jing XT, Xu M, Wan ZF and Zhang XY: miR-138 inhibits proliferation by targeting 3-phosphoinositide-dependent protein kinase-1 in non-small cell lung cancer cells. Clin Respir J 9: 27-33, 2015.

21. Xu R, Zeng G, Gao J, Ren Y, Zhang Z, Zhang Q, Zhao J, Tao H and Li D: miR-138 suppresses the proliferation of oral squamous cell carcinoma cells by targeting Yes-associated protein 1 . Oncol Rep 34: 2171-2178, 2015.

22. Liu X, Jiang L, Wang A, Yu J, Shi F and Zhou X: MicroRNA-138 suppresses invasion and promotes apoptosis in head and neck squamous cell carcinoma cell lines. Cancer Lett 286: 217-222, 2009.

23. Italiano A: Role of the EZH2 histone methyltransferase as a therapeutic target in cancer. Pharmacol Ther 165: 26-31, 2016.

24. Jang SH, Lee JE, Oh MH, Lee JH, Cho HD, Kim KJ, Kim SY, Han SW, Kim HJ, Bae SB and Lee HJ: High EZH2 protein expression is associated with poor overall survival in patients with luminal a breast cancer. J Breast Cancer 19: 53-60, 2016

25. Chen JF, Luo X, Xiang LS, Li HT, Zha L, Li N, He JM, Xie GF, Xie X and Liang HJ: EZH2 promotes colorectal cancer stem-like cell expansion by activating p21cip1-Wnt/ $\beta$-catenin signaling. Oncotarget 7: 41540-41558, 2016.

26. Yu W, Zhang G, Lu B, Li J, Wu Z, Ma H, Wang H and Lian R: MiR-340 impedes the progression of laryngeal squamous cell carcinoma by targeting EZH2. Gene 577: 193-201, 2016.

27. Zhang $\mathrm{H}$, Zhang H, Zhao M, Lv Z, Zhang X, Qin X, Wang H, Wang S, Su J, Lv X, et al: MiR-138 inhibits tumor growth through repression of EZH2 in non-small cell lung cancer. Cell Physiol Biochem 31: 56-65, 2013.

28. Qiu S, Huang D, Yin D, Li F, Li X, Kung HF and Peng Y: Suppression of tumorigenicity by microRNA-138 through inhibition of EZH2-CDK4/6-pRb-E2F1 signal loop in glioblastoma multiforme. Biochim Biophys Acta 1832: 1697-1707, 2013.

29. Liang J, Zhang Y, Jiang G, Liu Z, Xiang W, Chen X, Chen Z and Zhao J: MiR-138 induces renal carcinoma cell senescence by targeting EZH2 and is downregulated in human clear cell renal cell carcinoma. Oncol Res 21: 83-91, 2013.

30. Zhu Z, Tang J, Wang J, Duan G, Zhou L and Zhou X: MiR-138 acts as a tumor suppressor by targeting EZH2 and enhances cisplatin-induced apoptosis in osteosarcoma cells. PLoS One 11: e0150026, 2016 